The ASTROPHYSICAL JOURNAL, 612:000-000, 2004 September 10

(C) 2004. The American Astronomical Society. All rights reserved. Printed in U.S.A

\title{
SPECTRAL INDEX AND QUASI-PERIODIC OSCILLATION FREQUENCY CORRELATION IN BLACK HOLE SOURCES: OBSERVATIONAL EVIDENCE OF TWO PHASES AND PHASE TRANSITION IN BLACK HOLES
}

\author{
LeV TitarchuK ${ }^{1,2,3}$ aND RalPh FIORITo ${ }^{3,4}$ \\ Received 2004 February 18; accepted 2004 May 11
}

\begin{abstract}
Recent studies have shown that strong correlations are observed between the low frequencies (1-10 $\mathrm{Hz}$ ) of quasi-periodic oscillations (QPOs) and the spectral power law index of several black hole (BH) candidate sources, in low (hard) states, steep power law (soft) states, and transitions between these states. The observations indicate that the X-ray spectra of such state (phases) show the presence of a power-law component and are sometimes related to simultaneous radio emission, indicating the probable presence of a jet. Strong QPOs (>20\% rms) are present in the power density spectrum in the spectral range where the power-law component is dominant (i.e., $60 \%-90 \%$ ). This evidence contradicts the dominant, long-standing interpretation of QPOs as a signature of the thermal accretion disk. We present the data from the literature and our own data to illustrate the dominance of power-law index-QPO frequency correlations. We provide a model that identifies and explains the origin of the QPOs and how they are imprinted on the properties of the power-law flux component. We argue for the existence of a bounded compact coronal region that is a natural consequence of the adjustment of the Keplerian disk flow to the innermost sub-Keplerian boundary conditions near the central object and that ultimately leads to the formation of a transition layer (TL) between the adjustment radius and the innermost boundary. The model predicts two phases or states dictated by the photon upscattering produced in the TL: (1) a hard state, in which the TL is optically thin and very hot $(k T \gtrsim 50 \mathrm{keV}$ ), producing photon upscattering via thermal Comptonization (the photon spectrum index $\Gamma \sim 1.7$ for this state is dictated by gravitational energy release and Compton cooling in an optically thin shock near the adjustment radius), and (2) a soft state that is optically thick and relatively cold ( $k T \leqslant 5 \mathrm{keV}$; the index for this state, $\Gamma \sim 2.8$, is determined by soft-photon upscattering and photon trapping in a converging flow into the $\mathrm{BH}$ ). In the TL model for the corona, the QPO frequency $\nu_{\text {high }}$ is related to the gravitational (close to Keplerian) frequency $\nu_{\mathrm{K}}$ at the outer (adjustment) radius and $\nu_{\text {low }}$ is related to the TL's normal mode (magnetoacoustic) oscillation frequency $\nu_{\mathrm{MA}}$. The observed correlations between index and low and high QPO frequencies are readily explained in terms of this model. We also suggest a new method for evaluation of the BH mass using the index-frequency correlation.
\end{abstract}

Subject headings: black hole physics - stars: oscillations - X-rays: general

\section{INTRODUCTION}

The short dynamical timescales and the coherence of quasiperiodic oscillations (QPOs) observed in accreting X-ray bursts strongly suggest that QPOs can provide valuable information on the accretion dynamics in the innermost parts of these systems. In this respect the discovery of low $20-50 \mathrm{~Hz}$ QPOs in luminous neutron star (NS) binaries by van der Klis et al. (1985), kilohertz QPOs in NSs by Strohmayer et al. (1996), and hectohertz QPOs in black holes (BHs) by Morgan et al. (1997) opened a new era in the study of the dynamics near compact objects. Following these discoveries Psaltis et al. (1999) demonstrated that these NS and BH low and high frequencies follow a remarkably tight correlation. These features are the horizontal-branch oscillations (HBOs), along with the low-frequency noise Lorentzian $\nu_{\text {low }}$ and the lower $\mathrm{kHz}$ QPO $\nu_{\text {high }}$ for NS and $\mathrm{BH}$, respectively.

\footnotetext{
${ }^{1}$ George Mason University, School of Computational Sciences, Center for Earth Observing and Space Research, Fairfax, VA 22030.

${ }_{2}$ Naval Research Laboratory, Code 7655, Washington, DC 20375-5352; lev.titarchuk@nnl.navy.mil.

${ }^{3}$ NASA Goddard Space Flight Center, Code 660, Greenbelt, MD 20771; lev@lheapop.gsfc.nasa.gov.

${ }^{4}$ University of Maryland, College Park, MD 20742; rfiorito@umd.edu, rfiorito@milkyway.gsfc.nasa.gov.
}

Belloni et al. (2002) have updated the correlation of Psaltis et al. (1999) adding data from Nowak (2000), Boirin et al. (2000), Homan et al. (2001), Di Salvo et al. (2001), and Nowak et al. (2002). Psaltis et al. suggest that the low and high frequencies correlate in a way that seems to depend only weakly on the properties of the sources, such as the mass, the magnetic field, or possibly the presence of a hard surface in compact objects. Mauche (2002) has reported low- and highfrequency QPOs in the dwarf nova SS Cygni and in VW Hyi (see Wouldt \& Warner 2002 for the VW Hyi observations) and has called attention to the fact that these QPOs extend the correlation of Psaltis et al. downward in frequency by more than 2 orders of magnitude. This frequency correlation observed over such a broad range of sources indicates that a common phenomenon is responsible for QPOs in compact objects and raises the question of whether one can find an observational relation that would distinguish $\mathrm{BH}$ candidates from other compact sources.

The comparative study of spectral and QPO features in BH candidate sources has yielded important information that can help answer this question. As far as spectral properties are concerned, there is increasing observational evidence that the large complex of spectral states originally devised by Belloni et al. (2000) to classify the highly variable X-ray spectra of $\mathrm{BH}$ Galactic sources like GRS $1915+105$ can be reduced to a few 
simple configurations (see, e.g., McClintock \& Remillard 2003; Belloni 2003): (1) a low, hard power law-dominated state in which the photon spectral index is near $1.5 ;(2)$ a steep power law state in which the index is about 2.7 ; and (3) a thermally dominated state. The observed variability is explained by transitions between these three canonical configurations.

Low-frequency $(1-10 \mathrm{~Hz})$ QPOs are generally observed to be present in states 1 and 2 , during transitions between states 1 and 2, but never in state 3 . Both high- and low-frequency QPOs have been thought to be associated with the accretion disk, although their specific origin has not been widely understood (but see Titarchuk \& Wood 2002, hereafter TW02, for an explanation of their origin). Attempts to find consistent correlations with observable disk parameters have led to mixed results. In particular, there are so many exceptions to the observed correlations of frequency to disk spectral values sometimes observed that no general statement about the relation of the QPO with any spectral parameter solely associated with the disk, such as thermal flux, temperature, inner radius, etc., can be made. For example, exceptions to one of the most widely quoted correlations, i.e., between disk flux and frequency, have been presented for the well-studied microquasar GRS $1915+105$ by Fiorito et al. (2003) and for XTE $1550-564$ by Remillard et al. (2002a, 2002b).

On the other hand, a number of recent studies have revealed consistent and robust correlations between the photon index of the power-law component of the X-ray spectrum and low QPO frequency. Such strong correlations are observed the wellstudied BH binary GRS $1915+105$ in almost all situations in which the QPO is observable and in several other BHs over a wide range of observations and states.

These studies include the following:

1. A comprehensive study of correlations between photon index and QPO frequency in BH sources by Vignarca et al. (2003, hereafter V03); these results show strong correlations between index and QPO frequency, as well as saturation of the index between at the values $1.7 \pm 0.1$ and $2.7 \pm 0.2$ for several $\mathrm{BH}$ sources and for the same source in different states.

2. Recent studies by Kalemci (2002) for a number of Galactic $\mathrm{BH}$ X-ray transients observed during outburst decay that confirm the persistence of the correlation between frequency and index during state transitions.

3. Our own studies of GRS $1915+105$ on $\chi$ states (Fiorito et al. 2003), in which strong correlations of QPO frequency with power-law index have been observed; such states have traditionally been noted as low-brightness, power law-dominated

states (see, e.g., Belloni et al. 1999), where the disk is at least tenuous if not undetectable altogether.

4. Studies tracing the QPO frequency and index over time between state transitions show that the frequency tracks the index during state evolution (Homan et. al. 2001; Sobczak et al. 1999,2000 ) from a value of about $1.7 \pm 0.1$ to about $2.7 \pm 0.2$, as the QPO frequency varies from about 1 to $10 \mathrm{~Hz}$.

The persistence of the correlation of index with QPO frequency and its tracking with respect to time suggests that the underlying physical process or condition that gives rise to the low-frequency QPO are tied to the corona, which gives rise to the power-law component, and furthermore that this physical process or condition varies in a well-defined manner as the source progresses from one state to another. Moreover, the fact that the same characteristics of the correlation are seen in so many Galactic X-ray binary BH sources, which vary widely in both luminosity (presumably with mass accretion rate) and state, suggests that the physical processes controlling the index and the low-frequency QPOs are a characteristic property of these sources and, by virtue of the low-high frequency correlations of Psaltis et al. (1999) and Belloni et al. (2002), may be a universal property of all accreting compact systems.

The above data have motivated us to develop a detailed model of the physics of the corona surrounding a $\mathrm{BH}$ that directly predicts the behavior of the spectral index with fundamental properties of the corona. The model that we have developed incorporates fundamental principles of fluid mechanics, radiative transfer theory, and oscillatory processes. It identifies the origin of the QPO as a fundamental property of a compact coronal region near the $\mathrm{BH}$ and shows how the photon index of this corona changes as a function of mass accretion rate.

It has been already shown in a number of papers (see below) that the absence of a firm surface in BHs leads to the development of the very strong converging flow when the mass accretion rate is higher than Eddington rate $\dot{M}_{\text {Edd }}=$ $L_{\mathrm{Edd}} / \mathrm{c}^{2}$. The main observational features of the converging flow should be seen in high/soft phase, while the thermal Comptonization spectrum of the soft (presumably disk) photons should be seen in the hard phase.

In series of papers (Chakrabarti \& Titarchuk 1995; Titarchuk et al. 1996, 1997; Ebisawa et al. 1996; Titarchuk \& Zannias 1998; Shrader \& Titarchuk 1998, 1999; Borozdin Q3 et al. 1999; Laurent \& Titarchuk 1999, hereafter LT99; Laurent \& Titarchuk 2001; Titarchuk \& Shrader 2002; Turolla et al. 2002 ), the authors argue that the drain properties of the black $Q 4$ hole horizon are necessarily related to the bulk inflow in $\mathrm{BH} Q 5$ sources and that the spectral and timing features of this bulk inflow are rcally detected in X-ray observations of BHs. The signatures of the inflow are as follows:

1. An extended steep power law in which the photon index saturates to the value $2.7 \pm 0.2$ as the mass accretion rate increases; the precise value of the index is a function of the temperature of the flow, but its relatively high value is a result of inefficient photon upscattering in the converging flow due to photon trapping. LT99 demonstrated that the photon spectral index varies in a narrow range from 3 to 2.7 , with mass accretion rate (in Eddington units) increasing from 2 to 7. Moreover, LT99 demonstrated using Monte Carlo simulations that in the low/hard state when the plasma temperature is of order $50 \mathrm{keV}$ the bulk inflow spectrum is practically identical to the thermal Comptonization spectrum (there is no any noticeable effect of the bulk Comptonization in the spectrum). In fact, the effect of the bulk Comptonization compared to the thermal one becomes stronger when the plasma temperature drops below $10 \mathrm{keV}$. The small variation of the photon index around 1.7 is a characteristic signature of the low/hard state that was pointed out in earlier work by Chakrabarti \& Titarchuk (1995) and later confirmed by LT99.

2. The QPO high frequency $(100-300 \mathrm{~Hz})$, which is inversely proportional to $\mathrm{BH}$ mass. It is worth noting that the QPO frequency scaling as $1 / M$ is a generic feature of any QPO model in which QPO frequency is scaled with the Schwarzchild radius.

Furthermore, Titarchuk et al. (1999) presented observational evidence that the variability of the hard X-ray radiation in NSs and BHs occurs in a bounded configuration. Ford \& van der Klis (1998) found that the break frequency in the power density spectrum (PDS) is correlated with QPO frequency for some particular NS sources (4U 1728-32). Wijnands \& van der Klis 


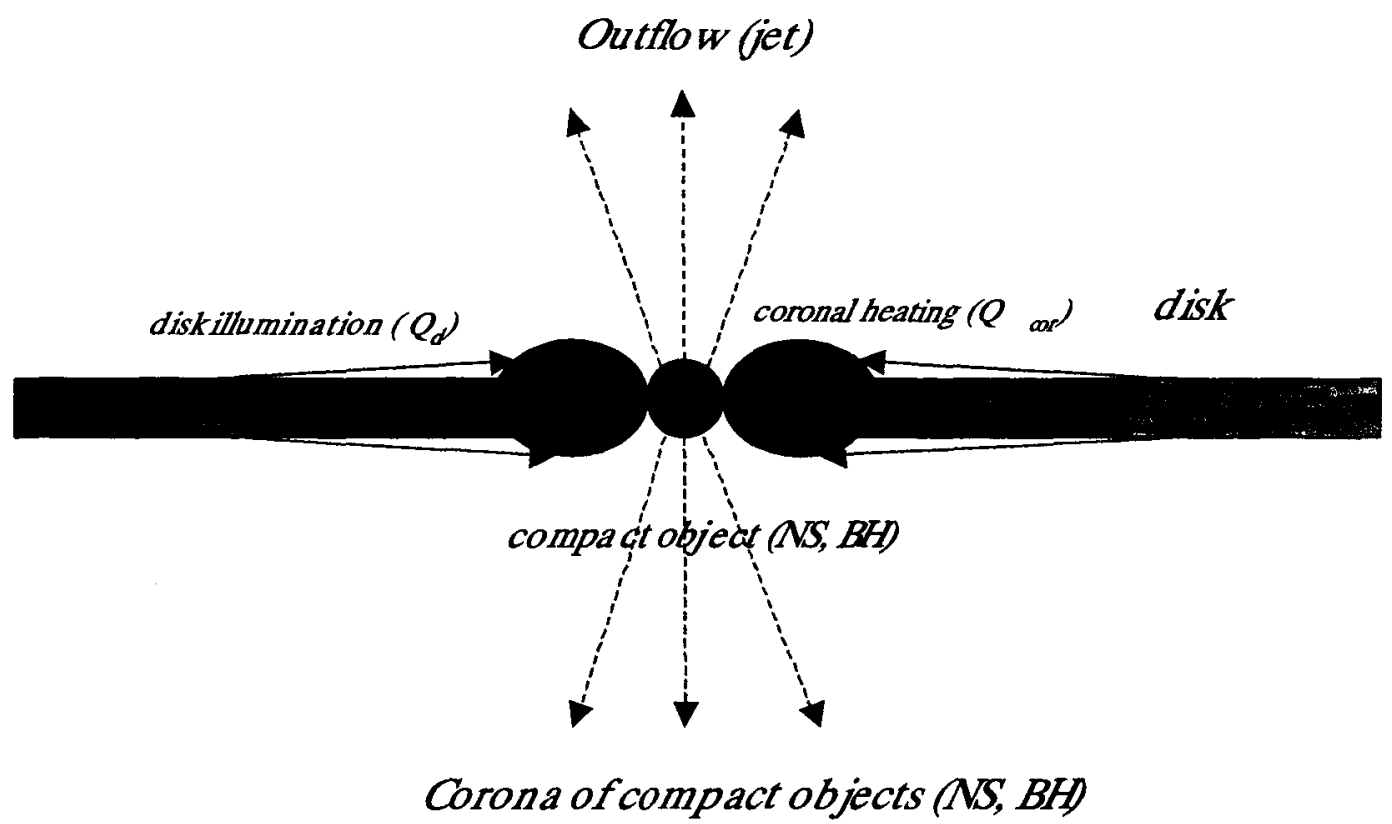

Fig. 1.-TL (corona) concept. This picture renders the gravitational energy release in the disk and corona and the coronal illumination by the disk soft flux in compact object (NS and BH). See text for details.

(1999) later found a similar correlation in BH sources. Titarchuk \& Osherovich (1999, hereafter TO99) and Titarchuk et al. (1999) explained this correlation in NSs and BHs, respectively. They argued that the observed PDS is the power spectrum of an exponential shot, $\exp \left(-t / t_{d}\right)$, which is the response of the diffusion propagation to any perturbation in a bounded medium. The inverse of the characteristic diffusion time $\nu_{b}=1 / t_{d}$ and the normal mode QPO frequency $\nu_{0}$ have a well-defined relation to the size of the bounded configuration, because $\nu_{b} \propto V / L$ and $\nu_{0} \propto V / L^{2}$, where $V$ is the specific perturbation (magnetosonic) velocity and $L$ is a characteristic size of the configuration (see TO99).

Titarchuk et al. (1998, hereafter TLM98) proposed that this bounded configuration (cavity) surrounding compact objects is the transition layer (TL) that is formed as a result of dynamical adjustments of a Keplerian disk to the innermost subKeplerian boundary conditions. They argued that this type of adjustment is a generic feature of the Keplerian flow in the presence of the sub-Keplerian boundary conditions near the central object and that it does not necessarily require the presence or absence of a hard surface. TLM98 concluded that an isothermal sub-Keplerian TL between the NS surface and its last Keplerian orbit forms as a result of this adjustment. The TL model is general and is applicable to both NS and BH systems. The primary problem in both NS and BH systems is understanding how the flow changes from pure Keplerian to sub-Keplerian as the radius decreases to small values. TLM98 suggested that the discontinuities and abrupt transitions in their solution result from derivatives of quantities such as angular velocities (weak shocks). They were the first to put forth the possibility of TL formation to explain most observed QFOs in bright low-mass X-ray binaries (LMXBs). In Figure 1 we illustrate the main idea of the TL concept for NSs and BHs.

In this paper we explain the general correlation between photon index and low frequency in the framework of the TL model and we give more arguments for the nature of the spectral phases (states) and phase transitions observed in BHs.
The main features of the TL model are given in $\S 2.1$. The formulation of the problem of low-frequency oscillations and the relationship with MA oscillations in the TL are also described in $\S 2.1$. The coronal model and the spectral indexoptical depth relation are given in $\S 2.2$. The derivation of the low frequency-index correlation and the details of modeling the spectral phase transition are presented in $\S 2.3$. We analyze specific QPO and spectral data in terms of the TL model in $\S 3$. We discuss the signatures and the methods of the identification of BH and NS sources using timing and spectral characteristics in $\S 4$. Our summary and conclusions follow in $\S 4$.

\section{TRANSITION LAYER THEORY}

\subsection{The Main Features of the Transition Layer Model}

TLM98 define the TL as a region confined between the inner sub-Keplerian disk boundary and the first Keplerian orbit (for the TL geometry, see Fig. 1 of this paper and Figs. 1 of TLM98 and Titarchuk et al. 1999). The main idea at the basis of our investigation is that a Keplerian accretion disk is forced to attain sub-Keplerian rotation close to the central object (a neutron star or a black hole). The transition between the Keplerian and the sub-Keplerian flow takes place in a relatively narrow, shocklike region (the size of the region if of order the radius of the central object [NS or BH]) where dissipation occurs. As a consequence, the gas temperature increases and the disk puffs up, forming a hot corona. Then the corona intercepts the soft disk photons and upscatters them via thermal and dynamical Comptonization, finally giving rise to the hard power law tail.

The puwer-láw index vahue strongingly depenends on the couronal optical depth and temperature (see Chakrabarti \& Titarchuk 1995; Titarchuk et al. 1997; LT99). When the mass accretion rate in the disk increases and consequently the disk soft photon flux increases, the corona is drastically cooled down to a temperature of order $5-10 \mathrm{keV}$ (in $\S 2.2$ we present a detailed analysis of this effect). For such a low plasma temperature the 
bulk motion of the converging flow is more efficient in upscattering disk photons than thermal Comptonization. Furthermore, the indices saturate to the asymptotic values around 2.7 as the mass accretion rate of the converging flow increases. The exact asymptotic value of the index is determined by the plasma temperature only (LT99).

TLM98 evaluate the size of the TL $L$ as a function of a nondimensional parameter $\gamma$ (often called the Reynolds number), which is the inverse of the $\alpha$-parameter for the accretion flow (Shakura \& Sunyaev 1973),

$$
\gamma=\dot{M} / 4 \pi \eta H=R V_{r} / D,
$$

where $\dot{M}=4 \pi R H m_{p} N_{e} V_{r}$ is the accretion rate in the disk, $H$ is a half-thickness of the disk, $V_{r}$ is a characteristic radial velocity at a given radius $R$ in the disk, $N_{e}$ is electron (proton) number density, $m_{p}$ is the proton mass, and $D$ is the diffusion coefficient. $D$ can be defined as $D=V_{t} l_{t} / 3$ using the turbulent velocity and the related turbulent scale, respectively, or as $D=D_{M}=c^{2} / \sigma$ for the magnetic case, where $\sigma$ is the conductivity (e.g., see details of the $D$ definition in Lang 1998). It is worth noting that the viscosity $\eta$ is a function of $\dot{M}$.

Because $\gamma$ and the TL Thomson optical depth $\tau_{0}$, which is a product of $L=R_{\text {out }}-R_{\text {in }}\left(R_{\text {in }}\right.$ and $R_{\text {out }}$ are the TL inner and the outer radius, respectively), electron number density $N_{e}$, and Thomson cross section $\sigma_{\mathrm{T}}\left(\tau_{0}=\sigma_{\mathrm{T}} N_{e} L\right)$, are both related to $\dot{M}$, one can in principle determine $\tau_{0}$ as a function of the $\gamma$-parameter. Because of the uncertainties of the disk equation of state and the viscosity $\eta$ in the disk, there is still uncertainty in the precise determination of this $\tau_{0}(\gamma)$ relation. We address this issue of the $\tau_{0}(\gamma)$ determination in $\S 2.3$.

TLM98 determine the $L(\gamma)$ dependence using the equation of motion in the disk where the radial motion is controlled by friction and the angular momentum exchange between adjacent layers, resulting in the loss of the initial angular momentum by accreting matter (e.g., Shakura \& Sunyaev 1973). We define an adjustment point where a Keplerian accretion disk is forced to attain sub-Keplerian rotation close to the central object (NS or BH). In order to determine the radius of the adjustment point one should combine this equation of motion with three boundary conditions: one at the innermost disk radius $R_{\text {in }}$ and two at the adjustment radius $R_{\text {out }}$. Namely, at $R_{\text {in }}$ the disk rotational frequency matches the rotational frequency of the central object (spin), and at $R_{\text {out }}$ there is no break in the rotational frequency, and its derivatives on the left-hand and right-hand sides are equal. Thus, for a given value of the $\gamma$-parameter the rotational frequency profile $\omega(R)$ and the outer radius of the TL are uniquely determined by the three boundary conditions and the equation of motion (see, for example, eqs. [8] and [9] of T099).

The adjustment of the Keplerian disk to the sub-Keplerian inner boundary creates conditions favorable for the formation of a hot plasma outflow at the outer boundary of the TL, because the Keplerian motion (if it is followed by sub-Keplerian motion) must pass through the super-Keplerian centrifugal barrier region (TLM98).

The equation (see TLM98, eq. [11], or TO99, eq. [9]) determines $R_{\text {out }}$ as a function of $\gamma$. For given values of $R_{\text {out }}(\gamma)$ one can find values of the Keplerian frequencies $\nu_{\mathrm{K}}\left(R_{\text {out }}\right)$ (see eq. [1] of TO99). For example, for the observed range of the high QPO frequencies in BH sources, from 1 to $100 \mathrm{~Hz}$ (see Fig. 2), one can find using the TLM98 solution for BH mass of $10 M_{\odot}$ that $\gamma$ varies from 2 to 20 (a variation that is related to the mass accretion rate $\dot{M}$; see eq. [1]). We remind the reader

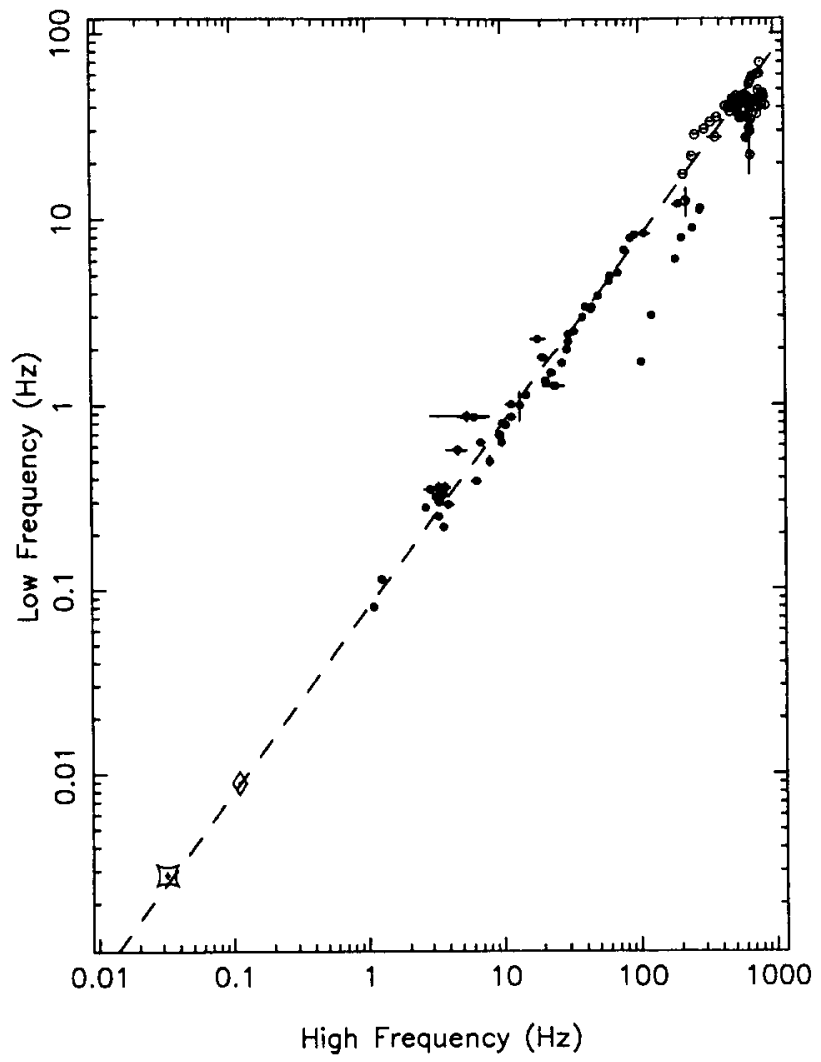

FIG. 2.-Correlation between frequencies of QPO and noise components for white dwarf (diamonds, SS Cyg [Mauche 2002]; squares, VW Hyi [Woudt \& Wamer 2002]), neutron star (open circles), and black hole candidate (filled circles) sources. Neution star and black hole data are from Belloni et al. (2002). The dashed line represents the best-fit of the observed correlation (see Titarchuk et al. 2001b and TW02, where this correlation is predicted and explained using the TL model). This plot also appears in Mauche (2002).

that in the framework of TLM98 these high QPO frequencies are interpreted as Keplerian frequencies $\nu_{\mathrm{K}}$ at $R_{\text {out }}$.

It is worth noting that in the literature $\alpha=\gamma^{-1}$ is treated as a constant and a free parameter independent of the mass accretion rate (or the spectral state), whereas we find that $\alpha$ varies from 0.5 to 0.05 with $\dot{M}$, i.e., that $\alpha$ is a very strong function of $\dot{M}$.

Soria (1999) inferred the effective value of the viscosity $\alpha_{\text {eff }}$ parameter as a function of the magnetic field. He showed that regardless of the true viscosity in the disk the $\alpha_{\text {eff }}$ parameter is always higher than 0.05 if the ratio of the magnetic pressure $P_{m}$ to the gas pressure $P_{g}$ is about $10 \%$ and more. Below (see also TW02 for details) we show, using the low frequencyhigh frequency correlation, that the inferred ratio of $P_{m} / P_{g}=$ $1 / \beta=0.1$ is consistent with values of $\alpha=0.05-0.5$ and Soria's predictions of high values of $\alpha$ at $P_{m} / P_{g} \gtrsim 0.1$.

The TL model identifies the low-frequency QPO as that associated with the viscous MA oscillation of the bounded TL; this mode is common to both BHs and NSs. The correlation of the MA frequency with the gravitational (Keplerian) frequency $\nu_{\mathrm{K}}=2 \pi \omega_{\mathrm{K}}$ was derived by TO99 and Titarchuk et al. (2001b) for NSs, and then it was generalized by TW02 for BHs and white dwarfs. The MA frequency is derived as the eigenfrequency of the boundary-value problem resulting from an MHD treatment of the interaction of the disk with the magnetic field. The problem is solved for two limiting boundary conditions that encompass realistic possibilities. The solution 


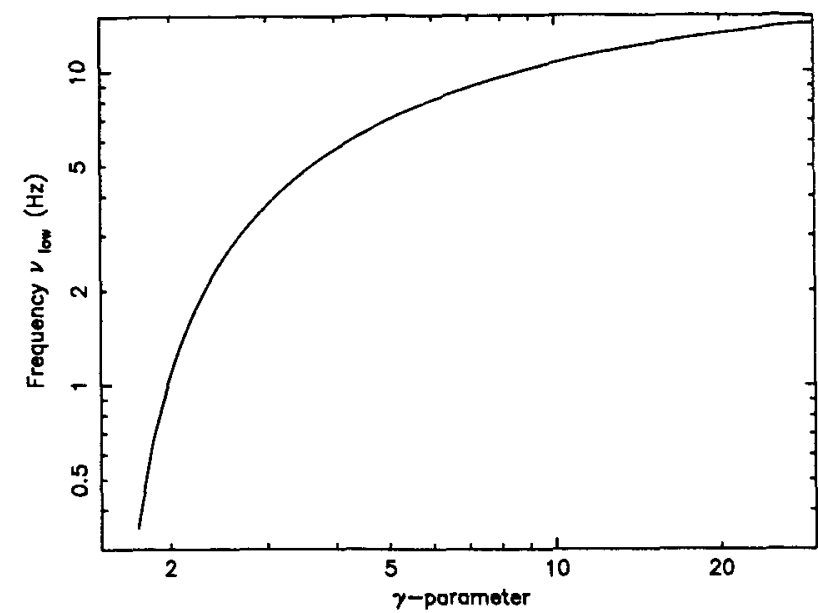

FIG. 3.-QPO low frequency vs. $\gamma$-parameter $(\propto \dot{M})$.

yields a velocity identified as a mixture of the sound speed and the Alfvén velocity. The Titarchuk et al. (2001b) treatment does not specify how the eigenfrequency is excited or damped. However, it makes clear that the QPO is a readily stimulated resonant frequency (see Titarchuk et al. 2002 and Titarchuk 2003 for details of excitation of the eigenfrequencies).

A linear relation was derived between $\nu_{\mathrm{MA}}$ and $\nu_{\mathrm{K}}$ (see Fig. 2 and TW02),

$$
\nu_{\mathrm{MA}}=C_{\mathrm{MA}} \nu_{\mathrm{K}}
$$

where $C_{\mathrm{MA}}=2^{1 / 2} 4 \pi[(1+f \beta) /(1+\beta)]^{1 / 2}\left(H / r_{\text {out }}\right)$ is a proportionality coefficient that is "universal" to the extent that $\beta$ and $H / R_{\text {out }}$ remain about the same from one source to the next. Here $\beta=P_{g} / P_{m}$ is the ratio of the gas pressure to the magnetic pressure, the coefficient $f=1 / 32$ and $1 / 32 \pi^{2}$ for stiff and free boundary conditions, respectively, and $H$ is the halfwidth of the Keplerian part of the disk.

The identification of $\nu_{\text {high }}$ and $\nu_{\text {low }}$ with $\nu_{\mathrm{K}}$ and $\nu_{\mathrm{MA}}$, respectively, leads TW02 to the determination of $H / R_{\text {out }}=$ $1.5 \times 10^{-2}$ and $\beta=10$ using the observed value of $C_{\mathrm{MA}}=$ 0.08 . On the other hand, $\nu_{\mathrm{K}}=\nu_{\text {high }}$ at the adjustment radius $R_{\text {out }}$ as a function of the $\gamma$-parameter can be readily obtained using equation (9) of TO99 [see above for details of $\nu_{\mathrm{K}}(\gamma)$ determination]. Thus, as a result of the identification of $\nu_{\text {low }}$ with $\nu_{\mathrm{MA}}$, we obtain the relation $\nu_{\text {low }}=\nu_{\mathrm{MA}}=C_{\mathrm{MA}} \nu_{\mathrm{K}}$ as a function of $\gamma$, namely,

$$
\nu_{\text {low }}=0.08 \nu_{\mathrm{K}}(\gamma)
$$

In Figure 3 we present the inferred $\nu_{\text {low }}$ as a function of $\gamma$. We derive this relation using equation (9) in TO99 with the assumptions that $a_{\mathrm{K}}=m\left(\nu_{0} / 363 \mathrm{~Hz}\right)=1$ (see eq. [7] of TO99 for definition $\left.\mathrm{a}_{\mathrm{K}}\right)$ and $m=M / M_{\odot}=10$.

It is evident that the inferred $\nu_{\text {high }}$ and $\nu_{\text {low }}$ are inversely proportional to $m$ because

$$
\begin{gathered}
\nu_{\text {high }}=\nu_{\mathrm{K}} \propto m^{-1}, \\
\nu_{\text {low }} \sim V / L
\end{gathered}
$$

where $L$ is the TL characteristic size, which is proportional to $m$.

We calculate the outer radius of the TL, $r_{\text {out }}=R_{\text {out }} / R_{S}$, as a function of $\nu_{\text {low }}$ using equation (2) or (3) above and

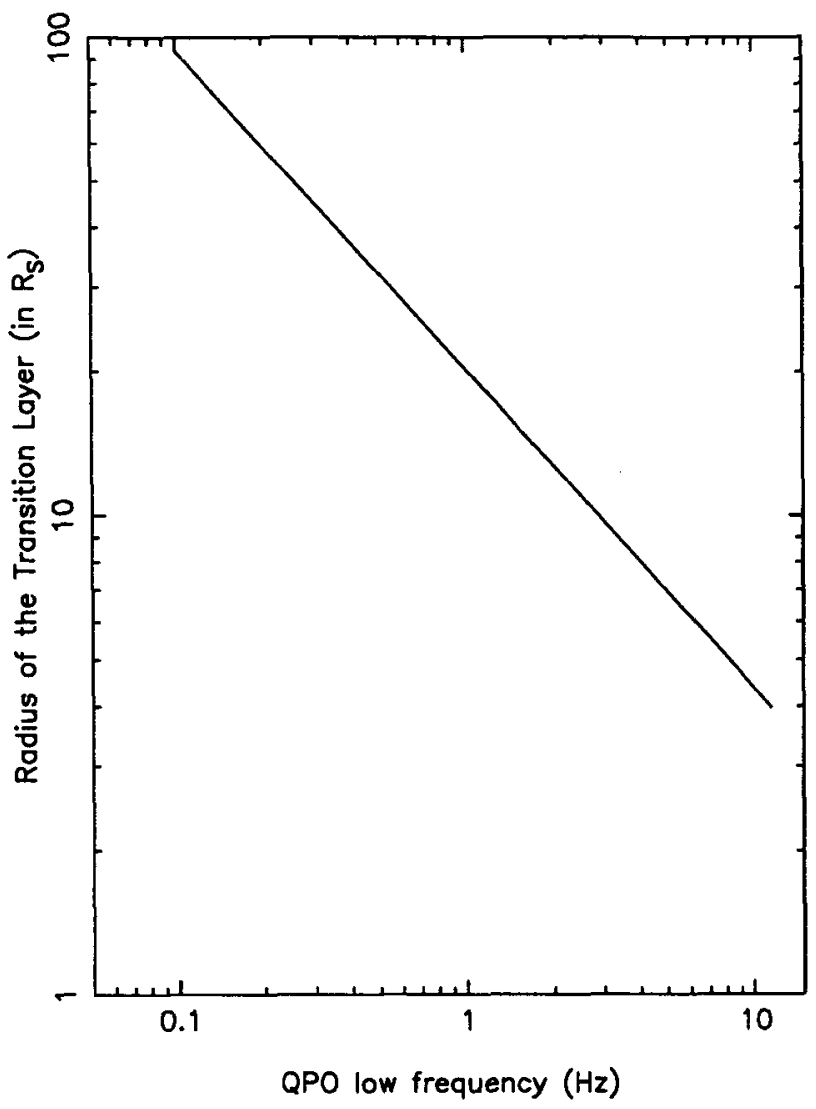

FIG. 4.-Outer (adjustment) radius of the TL vs. QPO low frequency.

equations (1) and (9) of TO99, keeping in mind that $\nu_{\text {high }}=$ $\nu_{\mathrm{K}}$. Because the radius of interest, which in our case is the outer TL boundary radius $R_{\text {out }}$, is related to $\nu_{\mathrm{K}}$, one can relate $R_{\text {out }}$ to $\nu_{\text {low }}$ using formula (3). We present this function in Figure 4.

The spectral index-frequency correlation can be derived if we find a spectral index-TL optical depth correlation. Because $\gamma$ is a function of the mass accretion rate $\dot{M}$ and the optical depth $\tau_{0}$ is a function of $\dot{M}$, we are led to a correlation of $\nu_{\text {low }}$ and $\tau_{0}$ and ultimately to the index-frequency correlation. It is worth noting that the $\gamma$ and $\tau_{0}$ dependences on $\dot{M}$ imply that a correlation between these quantities exists but do not imply that there is a linear correlation between $\gamma$ and $\tau_{0}$, because the $\gamma$-parameter also depends on the accretion flow viscosity $\eta$, which is also a function of $\dot{M}$.

\subsection{Spectral Index-Optical Depth Relation: Corona Model}

TLM98 offered the TL model adjustment of the Keplerian accretion flow (disk) to the sub-Keplerian inner boundary condition, which provides a compact corona for Comptonization upscattering of the illuminating accretion disk photons. The essentially sub-Keplerian rotation of NSs in LMXBs is a well-established observational fact due to the direct measurcments of NS spinins (sec a review by vāin der Klis 2000 for details). Direct BH spin measurements are still unavailable. Because the bulk motion spectral and timing model for a Schwarzschild black hole is probably consistent with observations (see Laurent \& Titarchuk 2001 for details), one can conclude that a fast-rotating Kerr $\mathrm{BH}$ is ruled out by the observations. In fact, there is no space between the inner edge 
of the disk and the horizon in the fast-rotating Kerr $\mathrm{BH}$ to upscatter the disk soft photons by the bulk motion to the energies of order $500 \mathrm{keV}$ that are detected in high-energy observations (Grove et al. 1998). Thus we can assume that BHs may also undergo sub-Keplerian rotation.

It is likely that this adjustment to sub-Keplerian flow is not smooth and that near the adjustment radius the strong or weak shocks can be formed. The adjustment radius (shock) region can be treated as a potential wall at which the accreting matter releases its gravitational energy in a geometrically thin target. As a consequence of this the shock plasma temperature is much higher than that in the surrounding regions of the Keplerian disk. The shock region gets puffed up oscillating with frequencies close to $\nu_{\mathrm{K}}$ (Titarchuk 2003). Additional oscillations can appear (at least in the NS case) because of the rotational configuration above the disk, which is a magnetosphere in the NS case (see details in Titarchuk et al. 1999). The shock formation leads to the formation of the hot inner bounded region. The hard photons in the shocked region illuminate the surrounding material, evaporating some part of the disk. It is obvious that the evaporated fraction depends on the mass accretion rate in the disk (see Chakrabarti \& Titarchuk 1995). Some small fraction of the hard photons (at a maximum of $6 \%$ ) can be reflected by the cold parts of the disk (Chakrabarti \& Titarchuk 1995; see also Basko et al. 1974).

Let us assume the column density of this region $y_{0}$ is a few grams or that the Thomson optical thickness $\tau_{0}$ is a few (see Shakura \& Sunyaev 1973 for the thickness estimate of the innermost part of the disk). The rate of energy release at this region $Q_{\text {cor }}$ is a few percent of the Eddington luminosity since the adjustment radius is located within 4-100 Schwarzschild radii (see Fig. 4). The heating of gas due to the gravitational energy release should be balanced by the photon emission. For high plasma temperature, Comptonization and free-free emission are the main cooling channels, and the heating of electrons is presumably due to the Coulomb collisions with protons (see Fig. 1 for a picture of the disk corona).

Under such physical conditions the energy balance can be written as (see also Zel'dovich \& Shakura 1969; TLM98)

$$
Q_{\mathrm{cor}} / \tau_{0}=C_{\mathrm{comp}} \epsilon(\tau) T_{e} / f\left(T_{e}\right)+C_{\mathrm{ff}} T_{e}^{1 / 2} \rho,
$$

where $\tau$ is the optical depth within the shock, $\epsilon(\tau)$ is a distribution function for the radiative energy distribution, $f\left(T_{e}\right)=$ $1+2.5\left(k T_{e} / m c^{2}\right)$ is the relativistic correction factor, $T_{e}$ is the electron temperature in kelvins, and $C_{\text {comp }}=20.2 \mathrm{~cm} \mathrm{~s}^{-1} \mathrm{~K}^{-1}$ and $C_{\mathrm{ff}}=2.6 \times 10^{20}$ ergs $\mathrm{K}^{-1 / 2} \mathrm{~cm} \mathrm{~s}^{-1}$ are dimensional constants. In this formula we neglect gas heating due to recoil effect.

The distribution $\epsilon(\tau)$ is obtained from the solution of the diffusion equation (Zel'dovich \& Shakura 1969),

$$
d^{2} \epsilon / d \tau^{2}=-3\left(Q_{\mathrm{tot}} / c\right) / \tau_{0}
$$

subject to two boundary conditions. Here we assume that the region of the gravitational energy release (corona) is a spherical shell surrounding the central object and the total flux in the corona is a sum $Q_{\mathrm{tot}}=Q_{\mathrm{cor}}+Q_{d}$ of the gravitational energy release $Q_{\text {cor }}$ and the illumination flux from outside of the corona (disk) $Q_{d}$. The inner and outer boundary condition for a $\mathrm{BH}$ corona is that there is no scattered radiation from outside of the corona. Then in the Eddington approximation (see Sobolev 1975) the boundary conditions can be written as follows:

$$
\left.(d \epsilon / d \tau-3 \epsilon / 2)\right|_{\tau=0}=\left.(d \epsilon / d \tau+3 \epsilon / 2)\right|_{\tau=\tau_{0}}=0,
$$

where $\tau=0$ and $\tau=\tau_{0}$ are at the inner and outer boundaries, respectively.

For an NS corona one should assume that inside of the inner corona boundary the energy density $\epsilon=$ constant, namely, that the radiation flux emitted toward the central object at some point of the inner boundary returns back (reflected by the NS surface); this condition is equivalent to the reflection condition:

$$
d \epsilon /\left.d \tau\right|_{\tau=0}=0
$$

Below we present a general formula of the coronal temperature that combines the $\mathrm{BH}$ and NS cases.

The solution of equations (7) and (8) provides us with the distribution function for the energy density in the BH corona:

$$
\epsilon(\tau)=\left(Q_{\text {tot }} / c\right)\left\{1+(3 / 2) \tau_{0}\left[\tau / \tau_{0}-\left(\tau / \tau_{0}\right)^{2}\right]\right\}
$$

We neglect the dependence of $Q_{\text {tot }}$ on $\tau$ to derive this formula. Furthermore, we take a representative value of $\langle\epsilon\rangle$ for the maximum value in the corona that is very close to the mean value of $\epsilon_{\text {mean }}$, i.e.,

$$
\langle\epsilon\rangle=\epsilon_{\max }=\left(Q_{\mathrm{tot}} / c\right)\left[1+(3 / 8) \tau_{0}\right]
$$

in order to approximate the coronal temperature. In Figure 5 we present the results of calculations of the temperatures $k T_{e}$ $(\mathrm{keV})$ and the energy spectral indices of the Comptonization spectrum $\alpha$ (photon index $\Gamma=\alpha+1$ ) as a function of the optical depth of the shell $\tau_{0}$. The different curves of $T_{e}\left(\tau_{0}\right)$ are related to the different ratios of $Q_{d} / Q_{\text {cor }}$. The calculations have been made for values of $\rho=10^{-6} \mathrm{~g} \mathrm{~cm}^{-3}$ and $Q_{\text {cor }}=10^{22} \mathrm{ergs}$ $\mathrm{cm}^{-2} \mathrm{~s}^{-1}$, which are characteristic values of the density and luminosity for the standard disk model (Shakura \& Sunyaev 1973). In fact, the calculation results weakly depend on $\rho$ and $Q_{\text {cor }}$ if

$$
\left(\rho / 10^{-6} \mathrm{~g} \mathrm{~cm}^{-3}\right) /\left(Q_{\text {cor }} / 10^{22} \operatorname{ergs~}^{-2} \mathrm{~s}^{-1}\right) \lesssim 1
$$

is of order a few or less. In this case the equation for the temperature is simplified

$$
6.73 \times 10^{-2} \tau_{0}\left(1+3 \tau_{0} / 8\right)\left(T_{e} / 10^{8} \mathrm{~K}\right)\left(1+Q_{d} / Q_{\mathrm{cor}}\right) / f\left(T_{e}\right)=1,
$$

which has the solution

$T_{e} / 10^{8} \mathrm{~K}=1.5\left[\tau_{0}\left(1+3 \tau_{0} / 8\right)\left(1+Q_{d} / Q_{\text {cor }}\right)-0.62\right]^{-1}$.

It is easy to show that the case for which $Q_{d} / Q_{\text {cor }}=1$ is identical to the NS case (the inner reflective boundary) when the disk illumination is neglected. One can see from equation (13) that $T_{e}$ is a function of $\tau_{0}$ if $Q_{d} / Q_{\text {cor }} \ll 1$. It is also worth noting that $T_{e} \tau_{0}\left(1+3 \tau_{0} / 8\right) / f\left(T_{e}\right)$ is insensitive to the total luminosity $Q_{\text {tot }}$ if $Q_{d} / Q_{\text {cor }} \ll 1$ (eq. [12]).

The spectral index $\alpha$ as a function of the product of $T_{e} \tau_{0}$ is insensitive to $Q_{\text {tot }}$ too for $\tau_{0} \lesssim 1$ because the Comptonization parameter

$$
y=4 k T_{e} / m_{e} c^{2} \max \left(\tau_{0}, \tau_{0}^{2}\right)
$$




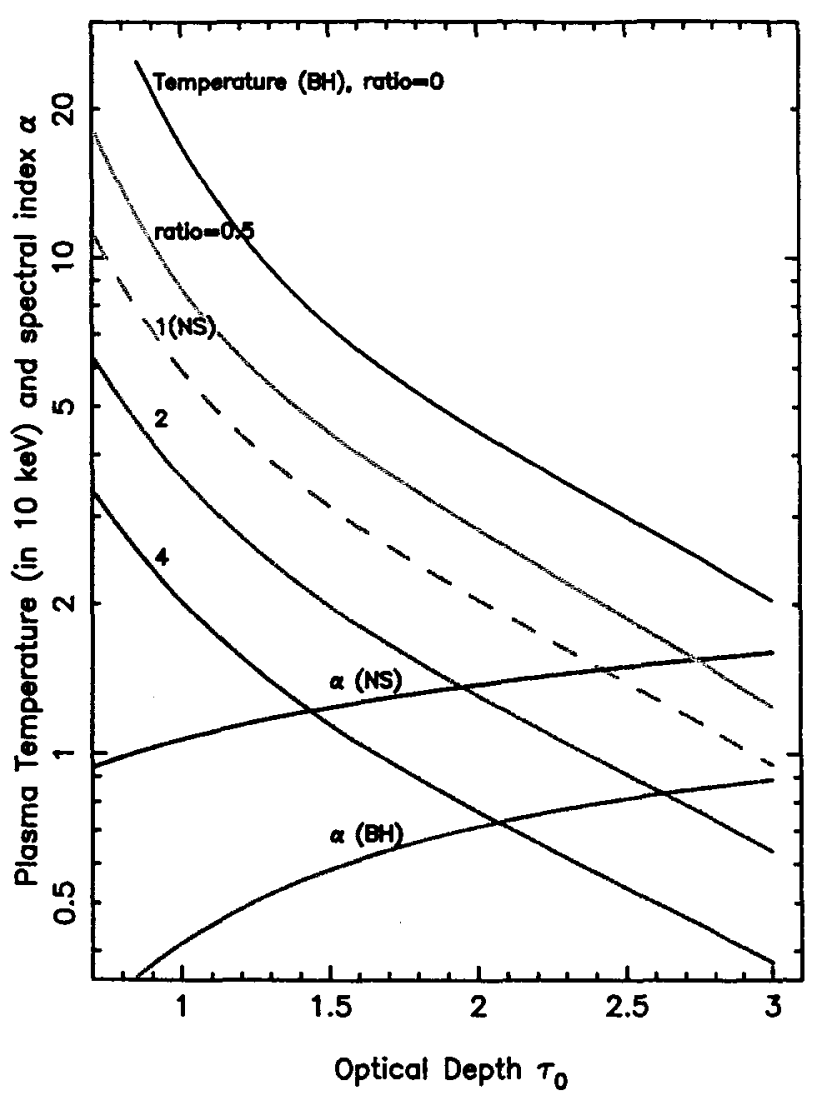

FIG. 5.-Energy spectral index vs. the TL optical depth (thermal Comptonization case).

and

$$
\alpha=-3 / 2+\sqrt{(9 / 4+4 / y)}
$$

(see Rybicki \& Lightman 1979; Sunyaev \& Titarchuk 1980). This inferred property of the Comptonization spectra reproduces the observed independence of $\alpha$ on the bolometric luminosity (see, e.g., Tanaka \& Lewin 1995) when $\alpha$ varies within the range $0.6 \pm 0.2(\Gamma=1.6 \pm 0.2)$. This defines the so-called low/hard state in BHs.

Below we show that the observed plateau of the indexfrequency correlation at low values of $\nu_{L}<1 \mathrm{~Hz}$ is the result of this behavior because the lower frequency values are related to the low mass accretion rates $\dot{m}=\dot{M} / M_{\text {Edd }}<1$ and $\tau_{0}$ is of order 1 when $\dot{m}<1$.

We calculate the exact values of the spectral indices $\alpha$ for a given $T_{e}$ and $\tau_{0}$ using the relativistic formulae developed by Titarchuk \& Lybarskij (1995). See their formulae (17) (for plane geometry; note that their $\tau_{0}$ is our $\left.\tau_{0} / 2\right)$ and (24). In Figure 5 we present the spectral indices as a function of $\tau_{0}$ for $\mathrm{BH}$ and NS cases when the disk illumination is negligibly small with respect to the coronal energy release (a condition that can be a modei for the low/hard state in these systems). The plasma temperature values of $20-150 \mathrm{keV}$ for $\tau_{0}=1-3$ and $\alpha=0.6 \pm 0.2$ (for ratio $=Q_{d} / Q_{\text {cor }}=0$ ) are typical values of these quantities for the low/hard state in BHs.

When $\mathrm{Q}_{d}$ is comparable to $Q_{\text {cor }}$, the cooling becomes more efficient because of Comptonization and free-free processes

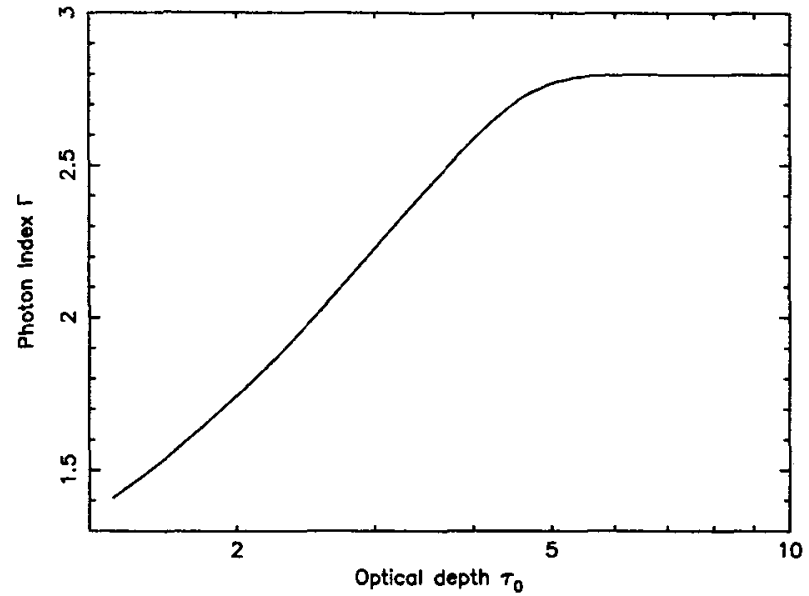

FiG. 6.-Photon spectral index vs. the TL optical depth (general case).

and therefore $T_{e}$ unavoidably decreases (see $T_{e}$ values for $Q_{d} / Q_{\text {cor }}=1-4$ in Fig. 5).

This illumination effect and mass accretion rate increase may explain the hard-soft transition when the Compton temperature drops substantially with increase of the soft (disk) photon flux (for illustration, see Fig. 1). In Figure 5 we show that the temperature drops from $50-60 \mathrm{keV}$ for ratio $=0$ to $7-$ $10 \mathrm{keV}$ for ratio $=4$. The temperature may also drop because of an increase in the optical depth (presumably because $\dot{m}$ increases) even when ratio $=0$.

\subsection{Modeling of Spectral Phase Transition from the Hard State to the Soft State}

LT99 studied the Comptonization of the soft radiation in the converging inflow (CI) into a BH using Monte Carlo simulations. The full relativistic treatment has been implemented to reproduce the spectra. LT99 show that spectrum of the soft state of BHs can be described as the sum of a thermal (disk) component and the convolution of some fraction of this component with the CI upscattering spread (Green's) function. The latter boosted photon component is seen as an extended power law at energies much higher than the characteristic energy of the soft photons. LT99 demonstrate the stability of the power-law index (the photon index $\Gamma=2.8 \pm 0.1$ ) over a wide range of the plasma temperature $0-10 \mathrm{keV}$ and mass accretion rates (higher than 2 in Eddington units) due to upscattering and photon trapping in the CI. The spectrum is practically the same as that produced by standard thermal Comptonization when the CI plasma temperature is of order $50 \mathrm{keV}$ (the typical ones for the BH hard state) and the photon index $\Gamma$ is around 1.7.

LT99 also demonstrate that the change of the spectral shapes from the soft state to the hard state is clearly related to the temperature and optical depth of the bulk inflow. We combine our results of calculations of index and Compton cloud temperatures for thermal Comptonization case (see $\S 2.2$ and Fig. 5) and LT99's results of the spectral calculations (see Table 2 of LT99 for the spectral index values) to evaluate the power-law index as a function of the optical depth of the Compton cloud $\tau_{0}$. We use the values of the index and of the temperature for $Q=0$ (see Fig. 5) when $\tau_{0}$ varies from 1 to 3 to describe the index behavior and the plasma temperature variation in the hard state. Figure 6 presents the photon index $\Gamma$ 
as a function of $\tau_{0}$. This function $\Gamma\left(\tau_{0}\right)$ can be fitted analytically as follows:

$$
\Gamma\left(\tau_{0}\right)= \begin{cases}c_{0}+c_{1} \tau_{0}+c_{2} \tau_{0}^{2}+c_{3} \tau_{0}^{3} & 1<\tau_{0} \leq 5.17 \\ b_{0}-b_{1} e^{-\tau_{0} / \tau_{*}} & \tau_{0}>5.17\end{cases}
$$

where $c_{0}=0.61, c_{1}=0.57, c_{2}=1.485 \times 10^{-2}, c_{3}=-8.5 \times$ $10^{-3}, b_{0}=2.81, b_{1}=1.0 \times 10^{3}$, and $\tau_{*}=0.5$.

In order to derive the index dependence $\Gamma$ on $\nu_{\text {low }}$, one should relate the parameter $\gamma$ to $\tau_{0}$ and then combine the two derived relationships $\Gamma$ versus $\tau_{0}$ and $\nu_{\text {low }}$ versus $\gamma$ (see Figs. 3-6). The optical depth $\tau_{0}$ and $\gamma$-parameter are functions of $\dot{M}$. To fit the data we assume that $\tau_{0}=A \gamma^{6}$. We have three free parameters, $\mathrm{BH}$ mass $m=M / M_{\odot}, \delta$, and $A$, to fit the model to the data if we assume that the $\mathrm{CI}$ is essentially cold (the CI temperature $k T_{\mathrm{ci}} \leqslant 5 \mathrm{keV}$ ). This relationship is all that is needed to fit the observed $\Gamma-\nu_{\text {low }}$ correlations.

\section{DATA AND ANALYSIS}

We analyze data points for photon index versus low frequency correlation obtained from Trudolyubov et al. (1999) and V03 for GRS $1915+105$ and Sobczak et al. $(1999,2000)$ for XTE J1550-564. Also, we make use of data for GRS $1915+105$ by Fiorito et al. (2003), which is a detailed study of QPO and spectral features of the steady low/hard states of GRS $1915+105$ for representative 1996 and 1997 observations. Fiorito et al. computed the power spectra of combined binned and event mode data for PCA channels 0-255 and sampled at $0.25-64 \mathrm{~Hz}$ for $4 \mathrm{~s}$ intervals. The spectra were averaged over the entire observation duration. The PDS spectra were fitted with a combination of Lorentzian lines and exhibited a break frequency, a central QPO frequency, and in some case the presence of the first harmonic. The background subtracted energy of each observation ID was obtained and time-averaged over the entire observation duration using standard 2 PCA and HEXTE data where available (both clusters). The XSPEC model used to fit the energy spectra consisted of a multitemperature thermal disk component (DISKBB), a simple power law, and a small iron line component. For the 1996 data (ObsSID 10408), it was found that the disk component could be neglected without affecting the fit. Acceptable $\chi^{2}$ values were obtained in all cases.

\subsection{Correlations between $Q P O$ Frequency and Spectral Parameters}

The first part of the Fiorito et al. (2003) study was to look for correlations of different spectral components, presumably thermal disk components, with QPO frequency to try to determine the reason for the nonunique total flux correlations previously observed. In the case of ObsID 20402 (1997), we did observe the expected positive correlation of QPO frequency with disk flux, in agreement with previous analyses of

Q10 Muno et al. (1999) and Markwardt et al. (1999), which were done in other states. However, we observed an anticorrelation of frequency with disk flux for ObsID 10408 (1996) and no correlation of frequency with disk flux for ObsID 10258 (1996). Furthermore, no correlation of QPO frequency with disk temperature is seen for any of the ObsIDS we studied. These results are at variance with the often-quoted result that disk parameters are closely associated with QPO frequency. Remillard et al. (2002b) show a comparison of low QPO frequency and the apparent disk flux for the source XTE J1550-564 during its 1998-1999 outburst. The QPOs are

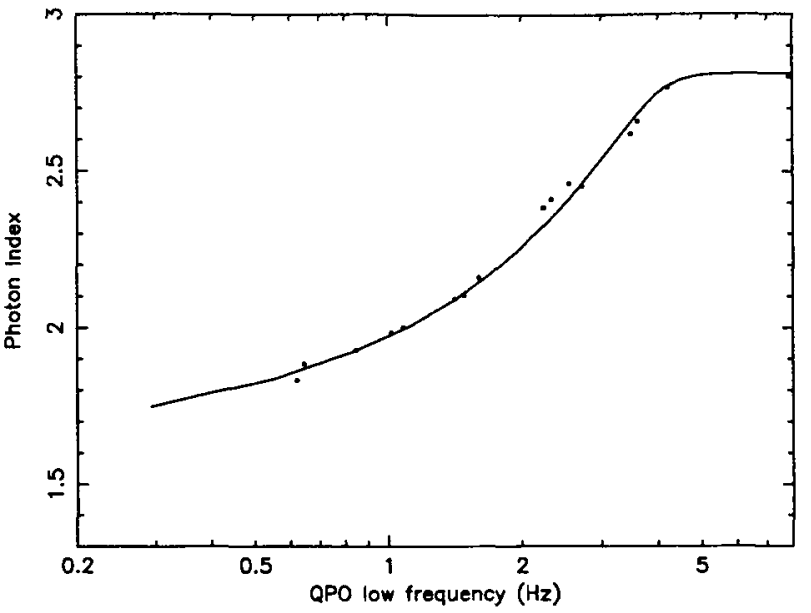

Fig. 7.-Power-law index vs. QPO centroid frequency for the plateau observations of GRS $1915+105$ from V03, along with a fit using the TL model with $m=12$ and $\tau_{0}=\gamma^{1.25}$.

reported with differences in phase lags. Again, it is clear that the frequency-disk flux correlation observed for this source is only observed at lower $(\nu<5 \mathrm{~Hz})$ QPO frequencies and fluxes than usually observed for GRS $1915+105$.

However, robust correlation between index and the central frequency of low-frequency QPOs is also seen in a wide variety of $\mathrm{BH}$ sources radiating in different states and during transition between states. The QPO frequencies in such studies are observed in the PDS obtained by integrating over time intervals ranging from the complete time of the observation to segmented time bins where the luminosity remains constant (see, e.g., Markwardt et al. 1999). Strong frequency-index correlations are observed by Kalemci (2002) in outburst decay (presumably state transitions) of a number of sources and by $\mathrm{V} 03$ in a comprehensive study that focuses its attention on the behavior of the power-law index and QPO frequency in several BH candidate sources. We will use the data of V03, our studies, and those of other studies as well in our analysis (see $\S 3.2$ below).

Observation of low-term tracking of the QPO frequency with the variations of the photon index has been observed in at least two BH sources. Tracking is seen in the data of Rossi (2003) and Homan (2001) in observations of XTE J1560-500 taken over a 30 day period. In this period the source apparently transits from the low/hard state $(\Gamma \sim 1.7)$ to the intermediate or soft state, where the index apparently remains close to the constant at a value $(\Gamma \sim 2.7)$. In this transition the $\mathrm{QPO}$ frequency varies from about 1 to $10 \mathrm{~Hz}$. Similar tracking is observed in XTE J1550-564 by Sobzcak et al. (1999, 2000). However, the index and QPO frequency does not track with the total X-ray flux (V03, Fig. 7).

\subsection{Comparison of the Inferred Index-Frequency Correlation with the Data: Low/Hard-High/Soft State Transitions}

\subsubsection{GRS $1915+105$}

In Figure 7 we present the results of fit to the data of V03 for the plateau observations of the microquasar GRS $1915+105$ using the TL model. The best-fit parameters are $m=12, \delta=1.25$, and $A=1$. We note that the value $m=12$ is consistent with the $\mathrm{BH}$ mass evaluation $m=13.3 \pm 4$ obtained by Greiner et al. (2001) using the IR spectra and by Shrader \& Titarchuk (2003; see also Borozdin et al. 1999) 


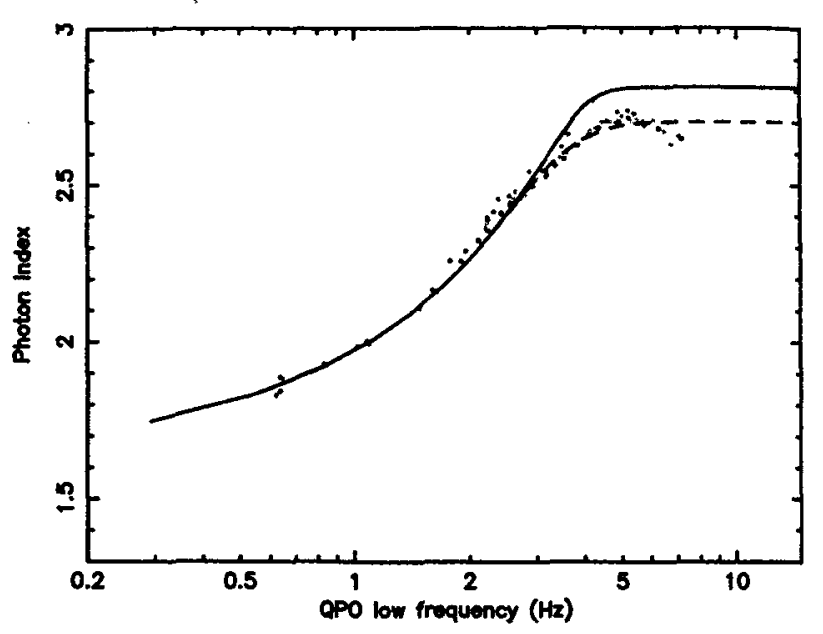

FIG. 8.-Power-law index vs. QPO centroid frequency for the observations of classes $\beta$ and $\nu$ (black points) and $\alpha$ and $\nu$ (red points; observations 15 and 16) of GRS $1915+105$ from V03, along with a fit using the TL model with $m=12$ and $\tau_{0}=\gamma^{1.25}$. Values for the plateau observations (see previous figure) are plotted for comparison (blue points).

using the X-ray spectra. The observable index saturations at low and high values of $\nu_{L}$ are nicely reproduced by the model. The low- and high-frequency plateau regions of the data and fit are signatures of the two spectral phases that are explained by two different regimes of Comptonization (upscattering): (1) bulk flow Comptonization in the soft state (saturation at $\Gamma \sim 2.8$ ) and (2) thermal Comptonization in the hard state (the photon index tends to level at $\Gamma \sim 1.7$ ).

A comparison of the index-frequency correlation of plateau observations with V03's $\beta$ and $\nu$ data (Belloni 1999 classifi-

Q11 cation scheme) and the model fits are plotted in Figure 8. The only difference in the data is different saturation values at high $\nu_{L}$, which can be readily explained by a change of plasma temperature in the converging inflow $k T_{\mathrm{ci}}$. For the upper value of $\Gamma=2.81$ the temperature $k T_{\mathrm{ci}}=5 \mathrm{keV}$, and for $\Gamma=2.7$ the temperature $k T_{\mathrm{ci}}=9 \mathrm{keV}$. The best-fit parameters for the latter data are the same as for the plateau observations, except that in the model function $\Gamma\left(\tau_{0}\right)$ (see eq. [14]) the coefficients $b_{0}$, $b_{1}$, and $\tau_{*}$ are replaced by $b_{0}=2.7, b_{1}=22.4$, and $\tau_{*}=0.8$ for $\tau_{0}>2.47$.

In Figure 9 we present a plot of power-law index versus QPO centroid frequency for the observations of class $\alpha$ and $\nu$ of GRS 1915+105 from V03 (black points; observation 18). Blue points correspond to the values for observations by Fiorito et al. (2003) for ObsIDs 20402 and 10258. Magenta points correspond to positions where the ObsID 20402 data points are shifted to one-half the measured QPO low-frequency values. This suggests that the latter data points are related to the second harmonics of the $\nu_{L}$ frequencies. It is well known that data for NS QPOs are a mixture of the first and the second harmonics of HBO frequencies (see, e.g., van der Klis 2000). Red points correspond to positions where there should be points with half of the frequencies for V03's observation 18. A theoretical curve (blue solid linc) obtaincd using $\Gamma\left(T_{0}\right)$ (see eq. [14]; for coefficients $b_{0}=2.7, b_{1}=22.4$, and $\tau_{*}=0.8$ for $\tau_{0}>2.47$ ) for $m=12$ and $\tau_{0}=\gamma^{1.5}$ fits Fiorito's ObsID 10258 data points.

It is worth noting that relation between $\gamma$ and $\tau_{0}$ is slightly different from what we obtained for the best fits presented in Figure 8. Namely, the index $\gamma$ increases from 1.25 to 1.5 . This

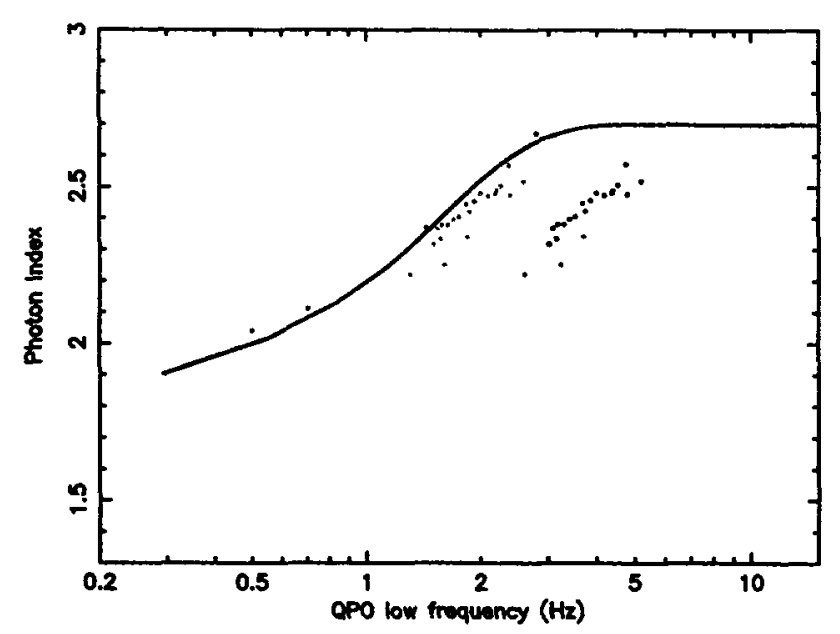

FIG. 9.-Power-law index vs. QPO centroid frequency for the observations of classes $\alpha$ and $\nu$ of GRS $1915+105$ from V03 (black points; observation 18). Blue points correspond to the values for observations by Fiorito et al (2003; see text). Magenta points correspond to positions where there should be points with half of those frequencies. Red points correspond to positions where there should be points with half of the frequencies for observation 18 . The blue solid curve is for $m=12$ and $\tau_{0}=\gamma^{1.5}$.

means that for the same value of $\gamma$ the optical depth $\tau_{0}$ is slightly higher than that in the state presented in Figure 8. This change can be explained by a slightly higher accumulation of plasma in the TL. For example, if we fix the disk viscosity $\eta$, then the $\gamma$-parameter is determined by $\dot{M}$ only. But the mass accretion rate $\dot{M}$ depends on the product of number density $N_{e}$ and the flow radial velocity $V_{r}$, i.e., $\dot{M} \propto N_{e} V_{r}$. Thus, for a given value of $N V_{r}$ the optical depth $\tau_{0} \propto N_{e}$ varies as $V_{r}$. Smaller values of $V_{r}$ corresponds to the higher values of $N_{e}$ and consequently to higher values of $\tau_{0}$; namely, higher plasma accumulation in the TL can be related to smaller velocities $V_{r}$.

It is interesting that the suggested half-frequency points (magenta and red points) are located in a narrow corridor between these inferred curves (presented in Figs. 8 and 9, respectively).

\subsubsection{XTE J1550-564}

Figure 10 shows a plot of power-law index versus QPO frequency for XTE J1550-564. All data points presented in Figures 10 and 11 (see also Figs. 6 and 8 of V03) were obtained by Sobczak et al. $(1999,2000)$ and Remillard et al. (2002a, 2002b)

The data again show a flattening out or saturation of the index at a value $\Gamma \sim 2.8$. It is clearly seen from Figure 8 of $V 03$ that most of the points presented there can be fitted by a smooth line except for a few points on the right that we temporarily exclude for our consideration. The shift transformation $\nu_{L}^{\prime}=$ $12 \nu_{L} / 10$ of the GRS $1915+105$ curve $\Gamma(\nu)$ (see Fig. 7) into $\Gamma\left(\nu^{\prime}\right)$ produces a fit for the XTE 1550-564 data. This means that if two sources are in the same accretion regime [i.e., when $\tau_{0}(\gamma)$ is the same], their relative $\mathrm{BH}$ masses can measured by the simpie shift transformation $\nu_{L}^{i}=m_{1} \nu_{L} / m_{2}$. In our case we use $m_{1}=12$ for GRS $1915+105$ and $m_{1}=10$ for XTE Q13 $1550-564$. These values are very close to the values that have been obtained by Shrader \& Titarchuk (2003) for these sources using X-ray spectroscopic methods. Thus the TL model provides an independent way to estimate the mass of one or more BHs in the same accretion state. 


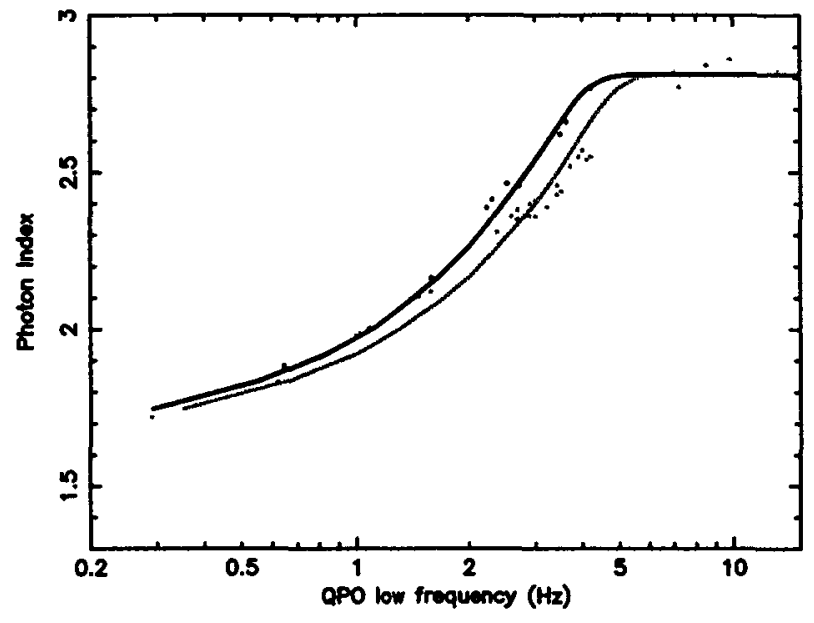

Fig. 10.-Comparison of the observed (points) and the theoretical correlations (curves) of photon index vs. QPO low frequency between GRS $1915+105$ (observations by V03) and XTE J1550-564 (observations by Sobczak et al. 1999, 2000; Remillard et al. 2002a, 2002b; see also Figs. 6 and 8 in V03). Black points and curve are for XTE J1550-564; red points and curve are for GRS $1915+105$. The XTE J1550-564 curve is produced by sliding the GRS 1915+105 curve along the frequency axis with factor $12 / 10$ (see text for details).

A comparison of all data points of correlating photon index versus QPO low frequency for XTE 1550-564 (black points) with the inferred correlation is shown in Figure 11. The saturation index value in the theoretical curve is related to the specific value of the plasma temperature of the converging inflow $k T_{\text {ci }}$, which changes from 5 to $20 \mathrm{keV}$ from the top curve to the bottom curve, respectively. Slightly different cooling regimes of the site of the converging bulk inflow can explain the different saturation levels of the index observed in XTE 1550-564. The temperature of the converging inflow is determined by the disk illumination geometry and by the intrinsic heating of the flow by pairs produced very close to the BH event horizon (see Laurent \& Titarchuk 2004). All these

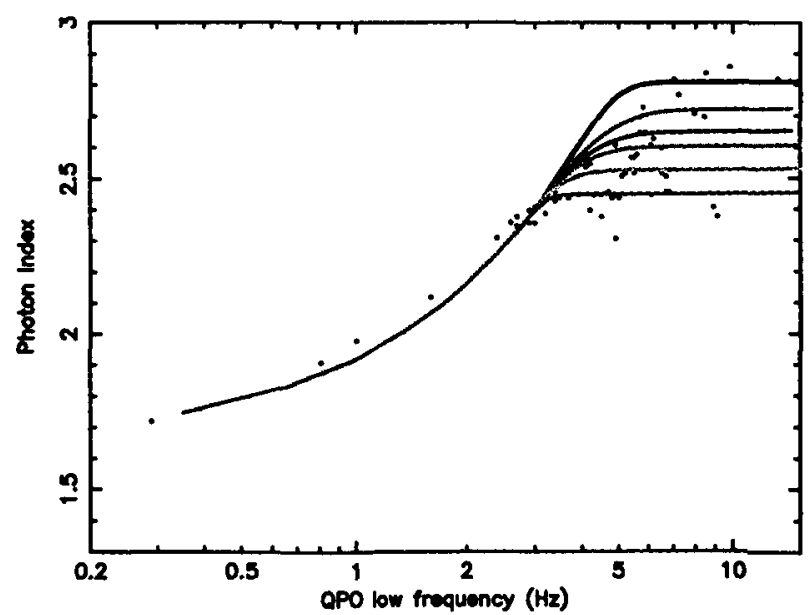

FIG. 11.-Comparison of the observed correlation of photon index vs. QPO low frequency for XTE $1550-564$ by Sobczak et al. $(1999,2000)$ and Remillard et al. (2002a, 2002b) (black points) with the inferred correlation. The saturation index value in the theoretical curve is related to specific value of the plasma temperature of the converging inflow $k T_{\mathrm{ci}}$, which changes from 5 to $20 \mathrm{keV}$ from the top curve to bottom.

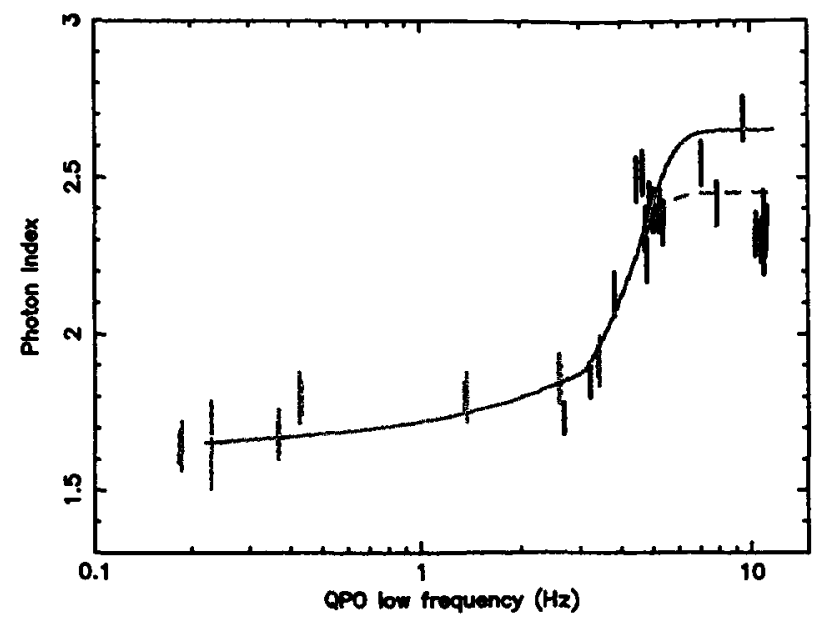

FIG. 12.-Comparison of the observed correlation of photon index vs. QPO low frequency for 4 U 1630-47 (Trudolyubov et al. 1999; Tomsick \& Kaaret 2000; Kalemci 2002) with the inferred correlation. The saturation index value in the theoretical curve is related to specific value of the plasma temperature of the converging inflow $k T_{\mathrm{ci}}$, which changes from 12 to $20 \mathrm{keV}$ from top to bottom.

effects may be manifested by the observed index-frequency correlation at high values of $\nu_{L}$.

\subsubsection{U $1630-47$}

Figure 12 shows a plot of index versus QPO frequency for 4U 1630-47 (Trudolyubov et al. 1999; Tomsick \& Kaaret 2000; Kalemci 2002). The data again show a similar behavior to that of XTE J1550-564 with an index saturation at $\Gamma=$ 2.45 and $\Gamma=2.65$. One can see two phases with the index level at $\Gamma=1.7 \pm 0.1$ and $\Gamma=2.6 \pm 0.1$ and the transition between them that is much sharper than that observed in the other sources discussed above. The best-fit curve $\Gamma\left(\nu_{L}\right)$ (solid line $)$ is obtained using $\tau_{0}=1.55 \gamma^{0.3}$ for $\gamma \leq \gamma=3.6$, $\tau_{0}=$ $1.55 \gamma_{*}^{-0.55} \gamma^{0.85}$ for $\gamma>\gamma_{*}$, and the $\Gamma\left(\tau_{0}\right)$ function (see formula [14]) for the best-fit $\mathrm{BH}$ mass $m=16$, which is close to the estimate made by Borozdin et al. (1999) using the X-ray spectroscopic method. The solid and dashed curves are for Q14 saturation index values $\Gamma=2.65$ (related to $k T_{\mathrm{ci}}=12 \mathrm{keV}$ ) and $\Gamma=2.45$ (related to $k T_{\mathrm{ci}}=20 \mathrm{keV}$ ), respectively. Coefficients $b_{0}=2.45$ and $2.65, b_{1}=53.7$ and 44.3 , and $\tau_{*}=$ 0.55 and 0.65 for $\tau_{0}>2.2$ and $\tau_{0}>3.42$, for the solid and dashed curves, respectively.

Our explanation for the observed anomalously sharp transition is as follows. Because the sharp transition QPO frequencies do not change we can speculate that the sharp TL size also does not change. But if the TL size is almost the same, then the $\gamma$-parameter is almost the same because the TL size depends on the $\gamma$-parameter only (see $\S 2.1$ ). But the spectral index is a function of $\tau_{0}$ and the TL plasma temperature. If the index rises drastically this means that upscattering is also drastically suppressed during the transition. Then we have only one possibility, i.e., that we are observing a sharp switching from the thermal Comptonization to the bulk motion Comptonization regimes. In the end of this transition we really see a signature of the bulk inflow in terms of the index saturation. The bulk motion Comptonization is dominant when the plasma temperature drops significantly to the value of order $10 \mathrm{keV}$. This can happen when a strong cooling emerges either because the optical depth $\tau_{0}$ increases because 
of the accumulation of matter in the TL or because an additional source of cooling such as a soft photon supply from the disk appears (see investigation of these cases in $\S 2.2$ ). The latter case can be realized if the corona intercepts more disk soft photons because of a puffing up of the disk or a build up of disk beneath the corona.

\section{DISCUSSION AND SUMMARY OF THE RESULTS}

We have developed a model of accretion onto a $\mathrm{BH}$ that greatly simplifies and reclassifies the plethora of observational "states" assigned to categorize the X-ray observations of variable $\mathrm{BH}$ candidates into two generic phases (states):

1. A soft state, in which we see the effect of the BH as a "drain." Bulk inflow upscattering and its quenching effect on the Comptonization of disk photons dominates the behavior of the BH spectrum. The power-law spectrum is steep in this situation. The observed high-energy photons are emitted from a compact region, where soft energy photons of the disk are upscattered by bulk matter inflow, forming the steep power law with photon index around 2.8, and low QPO frequencies (above $10 \mathrm{~Hz}$ ) and high QPO frequencies (of the order of $100 \mathrm{~Hz}$ ) are observed. In terms of the relativistic particle acceleration in the region of the corona and its effect on upscattering the soft disk photons, the soft-state spectrum is a result of the first-order Fermi acceleration with respect to $\mathrm{V} / \mathrm{c}$.

We would like to emphasize that bulk inflow is present in a $\mathrm{BH}$ when the mass accretion is high, but not in an $N S$, where the presence of the firm surface leads to a high radiation pressure, which eventually stops the accretion. The bulk inflow and all its spectral features are absent in NSs; in particular, the saturation of the index $\Gamma \sim 2.8$ with respect to QPO frequency, which is directly related to the optical depth and mass accretion rate, is observed in the soft spectral state of only BHs and is therefore a particular signature of a $\mathrm{BH}$.

2. A hard state, which is comparatively starved for accretion. The hard phase (state) is related to an extended thermal Compton scattering cloud (cavity) characterized by a photon index $\Gamma \sim 1.7$ and the presence of low QPO frequencies (below $1 \mathrm{~Hz}$ ). The low/hard spectrum is the result of a Fermi acceleration of the second order with respect to $(V / c)^{2}$. The effect of the first order on $V / c$ is smeared out by the quasi symmetry of the particular dynamic, predominantly thermal motion of the Compton cloud plasma.

The transition layer Comptonization model does the following:

1. It specifies the precise behavior of the power-law index for each state.

2. It identifies the origin and nature of low-frequency and high-frequency QPOs.

3. It predicts and explains the observed relationship between the QPOs and their dependence on mass accretion rate and spectral photon index for each of the phases.

4. It predicts and explains the observed robust correlation of low-frequency QPOs with the spectral index observed in BH candidates.

5. It predicts and explains the nbserved correlation between low- and high-frequency QPOs in BHs, NSs, and white dwarfs. We interpret the correlation between low frequency $\nu_{\text {low }}$ and power-law photon index $\Gamma$ in QPOs extensively investigated by Sobczak et al. $(1999,2000)$, Remillard (2000b), Kalemci (2002), and V03 for a variety of BH sources and states, and also by Homan et al. (2001) and Fiorito et al. (2003). The observed correlation strongly constrains theoretical models and provides clues to understanding the nature of the QPO phenomena, particularly in BHs and in compact objects in general.

The key assumptions and limitations of the model are as follows:

1. A sub-Keplerian rotation of central object (NS and BH), which is an observationally well established fact in NSs of LMXBs but still needs verification for BHs. However, the existence of an adjustment region, i.e., the TL, is a quite reasonable assumption for all but very rapidly rotating BHs. Unfortunately, up to now there has been no direct measurement of a BH spin.

2. Our fit to the data uses the model dependence of the index on the TL optical depth $\tau_{0}$ and the model dependence of the QPO frequencies on the $\gamma$-parameter. It is clear from the model that $\tau_{0}$ and $\gamma$ are correlated, but within the TLM98 model we cannot determine the exact $\tau_{0}(\gamma)$ dependence because of the uncertainty of the disk equation of the state and the viscosity in the disk. Therefore we have determined the $\tau_{0}(\gamma)$ relation by fitting the observed data, i.e., the correlation between the QPO low frequency and the photon spectral index. As a result of our analysis of the observed index-QPO correlation we conclude that the function $\tau_{0}(\gamma)$ is almost the same for GRS $1915+105$ and XTE J1550-564 but that it is drastically different for $4 U 1630-47$. For this source the inferred $\tau_{0}$ shows a very strong dependence of $\gamma$ on the QPO frequencies produced during a sharp spectral state transition. We speculate that this peculiar behavior of the index versus QPO frequency and consequently the inferred $\tau_{0}$ versus $\gamma$ is a result of the plasma accumulation in the TL. It is possible that a similar index-frequency correlation can be detected in other sources.

3. The model fits to the data give us the relatively low values of $\gamma=2-20$ (corresponding to viscosity parameter $\alpha=\gamma^{-1}$ from to 0.05 to 0.5 ). Such low values of $\gamma$ can be understood in the framework of MHD treatment of the disk viscosity. In fact, Soria (1999) inferred $\alpha$ of order 0.1 (regardless of the true disk viscosity) and higher if in the disk a magnetic pressure is $10 \%$ of the gas pressure or higher.

4. Within the TLM98 model one can estimate the absolute normalization of the magnetoacoustic (QPO low) frequencies (see, e.g., Titarchuk et al. 2001b), but a more precise normalization can only be obtained using the observed low-high frequency correlation (see Fig. 2).

5. In some very soft (or extended power law) regimes the positive correlation of frequency and index appears to be unbounded, i.e., there is no indication of saturation of the index value (Fiorito et al. 2003). Explanation for this effect is out of the scope of the present TL model. We can only suggest here that the extended power law regime occurs when strong outflows obscure the bulk inflow and the relatively cold outflow from winds downscatters the emerging high-energy photons and softens the observed spectrum (we will provide the details of this picture elsewhere).

6. V03's Figure 6 plots the index-frequency correlation for the two surces XTE Ji550-564 and GRO J1655-40 together. The behavior of the later sources is quite distinct from all others analyzed: it shows a reverse or negative correlation between photon index and frequency. This is the only case known to exhibit this type of behavior. We propose that at a high accretion rate regime the pair-production heating adds to and can dominate the heating in the cavity, causing a transition 
back to the thermal Comptonization phase. In this phase a reverse correlation between low QPO frequency can result and is our explanation of the single case where this phenomena is observed. Further theoretical investigation of this case will be addressed in future studies.

The main results of this paper are as follows:

1. We find that the observed low frequency-index correlation is a natural consequence of an adjustment of the Keplerian disk flow to the innermost sub-Keplerian boundary conditions near the central object. This ultimately leads to the formation of the sub-Keplerian transition layer between the adjustment radius and the innermost boundary (the horizon for $\mathrm{BH}$ ).

2. In the framework of the TL model, $\nu_{\text {high }}$ is related to the gravitational frequency at the outer (adjustment) radius $\nu_{g} \approx \nu_{\mathrm{K}}$ and $\nu_{\text {low }}$ is related to the magnetoacoustic oscillation frequency

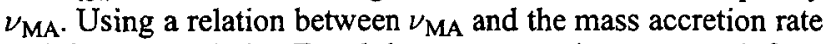
and the photon index $\Gamma$ and the mass accretion rate, we infer a correlation between $\nu_{\mathrm{MA}}$ and the spectral index $\Gamma$.

3. The identification of $\nu_{\text {low }}$ with $\nu_{\mathrm{MA}}$ allows us to make a comparison of the theoretically predicted correlation with the observed correlation. For this identification we use the singletemperature plasma assumption. We apply the plasma temperature obtained from the Comptonization spectra (electron temperatures) for calculations of magnetoacoustic frequencies, which strongly depends on the proton temperature. The single-temperature assumption is really consistent with the data.

4. We present strong arguments that in BHs two particular distinct phases occur, where one of them, the steep power law phase, is a real $\mathrm{BH}$ signature.

5. We found that the hard phase (state) is related to an extended Compton cloud (cavity) characterized by a photon index of around 1.7 and low QPO frequencies below $1 \mathrm{~Hz}$. This is the regime where thermal Comptonization dominates the upscattering of soft disk photons and the spectral shape (index) is almost independent of mass accretion rate.

6. We find that the soft phase (state) is related to the very compact region where soft energy photons of the disk are upscattered, forming a steep power law spectrum with the photon index saturating around 2.8. This is the regime where bulk flow Comptonization dominates and the effect of an increase in the mass accretion is offset by the effect of photon trapping in the converging flow into the $\mathrm{BH}$.

7. In the context of the distinction between NS and $\mathrm{BH}$ sources, it is worth pointing to a certain situation that can occur in low/hard states. The source should be an NS if the soft blackbody-like component with a color temperature of order $\mathrm{keV}$ is present in the spectrum (Torrejon et al. 2004). In contrast, such a high temperature is necessarily related to the high disk luminosity in the $\mathrm{BH}$ case.

8. We offer a new method of BH mass estimate using the index-frequency correlations. Namely, if the theoretical curve of the index-frequency dependence $\Gamma\left(\nu_{L}\right)$ related to the parameter $m_{1}$ fits the data for a given source, then the simple slide of the frequency axis $\nu_{L}^{\prime}=\left(m_{1} / m_{2}\right) \nu_{L}$ with respect to $\nu_{L}$ may allow us to obtain the mass $m_{2}$ by the fit of $\Gamma\left(\nu_{L}^{\prime}\right)$ to the observable correlation for another source.

We acknowledge Tomaso Belloni for kindly supplying us with the data published in V03. We gratefully appreciate the referee's suggestions for improving and clarifying our results and the limitations of our model.
Basko, M. M., Sunyaev, R. A., \& Titarchuk, L. 1974, A\&A, 31, 249 Belloni, T. 1999, in Highlights in X-Ray Astronomy, ed. B. Aschenbach \& M. J. Freyberg (MPE Rep. 272; Garching: MPI), 82

. 2003, preprint (astro-ph /0309028)

Belloni, T., Méndez, M., van der Klis, M., Lewin, W. H. G., \& Dieters, S. 1999 , ApJ, 519, L159

Belloni, T., Psaltis, D., \& van der Klis, M. 2002, ApJ, 572, 392

Belloni, T., et al. 2000, A\&A, 355, 271

Boirin, L., Barret, D., Olive, J. F., Bloser, P. F., \& Grindlay, J. E. 2000, A\&A, 361,121

Borozdin, K, et al. 1999, ApJ, 517, 367

Chakrabarti, S. N., \& Titarchuk, L. 1995, ApJ, 455, 623

Di Salvo, T., Méndez, M., van der Klis, M., Ford, E., \& Robba, N. R. 2001, ApJ, 546, 1107

Ebisawa, K., Titarchuk, L., \& Chakrabarti, S. 1996, PASJ, 48, 59

Fiorito, R., Markwardt, C., \& Swank, J. 2003, BAAS, 35, 11.07

Ford, E., \& van der Klis, M. 1998, ApJ, 506, L39

Greiner, J., Cuby, J.-G., \& McCaughrean, M. J. 2001, Nature, 414, 522

Grove, J. E. 1998, ApJ, 500, 899

Homan, J., et al. 2001, ApJS, 132, 377

Kalemci, E. 2002, Ph.D. thesis, UC San Diego

Lang, K. R. 1998, Astrophysical Formulae (Berlin: Springer)

Laurent, P., \& Titarchuk, L. 1999, ApJ, 511, 289 (LT99)

- 2001, ApJ, 562, L67

Q15

2004, in preparation

Markwardt, C., et al. 1999, ApJ, 513, L37

Mauche, C. 2002, ApJ, 580, 423

McClintock, J., \& Remillard, R. 2003, preprint (astro-ph/0306213)

Morgan, E. H., Remillard, R. A., \& Greiner, J. 1997, ApJ, 482, 993

Muno, M. P., Morgan, E. H., \& Remillard, R. A. 1999, ApJ, 527, 321

Nowak, M. A. 2000, MNRAS, 318, 361

Nowak, M. A., Wilms, J., \& Dove, J. B. 2002, MNRAS, 332, 856

Psaltis, D., Belloni, T., \& van der Klis, M. 1999, ApJ, 520, 262

Remillard, R., et al. 2002a, ApJ, 580, 1030

\section{REFERENCES}

Remillard, R, et al. 2002b, ApJ, 564, 962

Rossi, S., et al. 2003, preprint (astro-ph/0309129)

Rybicki, G. B., \& Lightman, A. P. 1979, Radiative Processes in Astrophysics (New York: Wiley)

Shakura, N. I., \& Sunyaev, R. A. 1973, A\&A, 24, 337

Shrader, C., \& Titarchuk, L. 1998, ApJ, 499, L31

1999, BAAS, 31, 731

2003, ApJ, 598, 168

Sobczak, G. J., et al. 1999, ApJ, 517, L121 2000, ApJ, 531, 537

Sobolev, V. V. 1975, Light Scattering in Planetary Atmospheres (Oxford: Pergamon)

Soria, R. 1999, Ph.D. thesis, Australian National Univ., Canberra

Strohmayer, T. E., et al. 1996, ApJ, 469, L9

Sunyaev, R., \& Titarchuk, L. 1980, A\&A, 86, 121

Tanaka, Y., \& Lewin, W. H. G. 1995, in X-Ray Binaries, ed. W. H. G. Lewin J. van Paridis, \& E. P. J. van den Heuvel (Cambridge: Cambridge Univ Press), 126

Titarchuk, L. 2003, ApJ, 591, 354

Titarchuk, L., Bradshaw, C. F., Geldzahler, B. J., \& Fomalont, E. B. 2001a ApJ, 555, L45

Titarchuk, L., Bradshaw, C. F., \& Wood, K. S. 2001b, ApJ, 560, L55

Titarchuk, L., Cui, W., \& Wood, K. 2002, ApJ, 576, L49

Titarchuk, L., Lapidus, I., \& Muslimov, A. 1998, ApJ, 499, 315 (TLM98)

Titarchuk, L., \& Lybarskij, Yu. 1995, ApJ, 450, 876

Titarchuk, L., Mastichiadis, A., \& Kylafis, N. 1996, A\&AS, 120, 171 1997, ApJ, 487, 834

Titarchuk, L., \& Osherovich, V. 1999, ApJ, 518, L95 (TO99)

Titarchuk, L. Osherovich, V., \& Kuznetsov, S. 1999, ApJ, 525, L129

Titarchuk, L., \& Shrader, C. 2002, ApJ, 567, 1057

Titarchuk, L., \& Wood, K. 2002, ApJ, 577, L23 (TW02)

Titarchuk, L., \& Zannias, T. 1998, ApJ, 493, 863

Tomsick, J. A., \& Kaaret, P. 2000, ApJ, 537, 448

Torrejon, J. M., et al. 2004, A\&A, in press 
Trudolyubov, S. P., Churazov, E. M., \& Gilfanov, M. R. 1999, Astron. Lett., 25,718

Turolla, R., Zane, S., \& Titarchuk, L. 2002, ApJ, 576, 349

van der Klis, M. 2000, ARA\&A, 38, 717

van der Klis, M., et al. 1985, Nature, 316, 225
Vignarca, F., Migliari, S., Belloni, T., Psaltis, D., \& vander Klis, M. 2003, A\&A, 397, 729 (V03)

Wijnands, A. D., \& van der Klis, M. 1999, ApJ, 514, 939

Woudt, P. A., \& Wamer, B. 2002, MNRAS, 333, 411

Zel'dovich, Ya. B., \& Shakura, N. I. 1969, Soviet Astron., 13, 175 


\section{AUTHOR QUERIES}

Q1 Auth: Your article has been edited for grammar, consistency, and to conform to ApJ joumal style (see Instructions to Authors). To expedite publication, we generally do not query every routine grammatical and style change made to a manuscript, although all substantive changes have been noted. Please review the article carefully. Note that we may be unable to make changes that conflict with journal style, obscure meaning, or create grammatical or other problems. It is ApJ style to have no titles on figures. Any text appearing at the top of a figure, with the exception of an axis label, has been removed and incorporated into the legend. Also note that article proofs via PDF do not show corrections that will have been made by the typesetter's proofreader, which generally include corrections of errors of page layout, figure placement, and spacing and font mistakes; feel free to mark any errors you notice in these areas. ${ }^{* *}$ When sending us your corrections, if you are writing them by hand, please print clearly. If sending a fax, please do not write too close to the margins of the page, since these are often cut off in fax transmission. Finally, please note that a delayed, incomplete, or illegible response may delay the publication of your article while we contact you.**

Q2 Auth: Please confirm or correct the reference I have supplied for Belloni et al. 1999.

Q3 Auth: Please confirm or correct the reference I have supplied for Shrader \& Titarchuk 1999.

Q4 Auth: Please confirm or correct the reference I have supplied for Turolla et al. 2002.

Q5 Auth: Changed "black horizon" to "black hole horizon". OK?

Q6 Auth: Changed Titarchuk 2002 to 2003 to match reference.

Q7 Auth: ApJ style uses $\epsilon$ rather than $\varepsilon$ unless both are needed in a paper. I have changed $\varepsilon$ to $\epsilon$ throughout.

Q8 Auth: Added Lewin to Tanaka 1995 to match entry in reference list.

Q9 Auth: Changed "black mass" to "BH mass". Is this correct?

Q10 Auth: Please confirm or correct the reference I have supplied for Muno et al. 1999.

Q11 Auth: Please confirm or correct the reference I have supplied for Belloni 1999.

Q12 Auth: There is no eq. (17) in this paper. I have changed the references to eq. (17) here and below to eq. (14). Is this correct?

Q13 Auth: Changed $m=10$ to $m_{1}=10$. Is this correct?

Q14 Auth: Please ensure that the long sentence beginning "The best-fit curve ..." is OK as edited.

Q15 Auth: Has article been submitted yet? If so, to which journal? If not yet submitted, entry will be removed from reference list, and citations in text will indicate "in preparation".

Q16 Auth: Please cite or delete reference. 